\title{
Characteristics of Functional Components and Antioxidant Activity of 28 Common Beans
}

\author{
Chengyu Jiang ${ }^{1,2}$, Zhaohong $\mathrm{Ci}^{1,2}$, Shuo Feng ${ }^{1,2}$, Shan $\mathrm{Wu}^{1,2}$, Michiyuki Kojima ${ }^{1{ }^{1 *}}$ \\ ${ }^{1}$ Department of Food Science, Graduate School of Animal Husbandry, Obihiro University of Agriculture and Veterinary Medicine, \\ 080-8555, 11, Nishi-2-Sen, Inada-Cho, Obihiro, Hokkaido, Japan. \\ ${ }^{2}$ Department of Bioresources Science, United Graduate School of Agricultural Sciences, Iwate University, 3-18-8, \\ Ueda, Morioka, Iwate 020-8550, Japan \\ *Corresponding author: kojima@obihiro.ac.jp
}

\begin{abstract}
Common beans are either white or colored. They contain large amounts of phenolic compounds and other phytochemicals. Polyphenols exhibit high antioxidant activity that promote health by reducing oxidative stress. The objective of this study was to compare the content and composition of polyphenols in 28 common beans and determine the relation between their antioxidant activities and seed coat color. Here, we measured seed coat color by the International Commission in Illumination method, estimated polyphenol content and antioxidant activity by colorimetry, and identified polyphenol compositions by RP-HPLC. The results showed that polyphenol content and antioxidant activity were higher in colored beans than in the white beans. Taisho-Kintoki (red kidney) had the highest polyphenol content ( $6.12 \mathrm{mg}$ / g seed) and antioxidant activity $(21.98 \mu \mathrm{mol} / \mathrm{g}$ seed; $3.75 \mathrm{mg} / \mathrm{g}$ seed). There was a high correlation between the total polyphenol content and seed coat redness. There were also high equilateral correlations between antioxidant activity and polyphenol content. Twelve phenolic compounds were identified. Based on their polyphenol compositions synthesized by various enzymes, the 28 common beans were divided into three groups. White beans and several half-spotted beans contained a large amount of catechin-7-O-glucoside with low antioxidant activity. Anthocyanin, procyanidin, and kaempferol-3-O-glucoside constituted significant proportions of the total polyphenol compounds and were positively correlated with antioxidant activity in the colored common beans. These findings are expected to help guide consumers and breeders in the selection of common bean varieties with high antioxidant activity.
\end{abstract}

Keywords: common bean, polyphenols, antioxidant activity, principal component analysis

Cite This Article: Chengyu Jiang, Zhaohong $\mathrm{Ci}$, Shuo Feng, Shan $\mathrm{Wu}$, and Michiyuki Kojima, "Characteristics of Functional Components and Antioxidant Activity of 28 Common Beans." Journal of Food and Nutrition Research, vol. 6, no. 7 (2018): 439-444. doi: 10.12691/jfnr-6-7-3.

\section{Introduction}

In Japan, the common bean is mostly cultivated in Hokkaido. The beans are categorized as either white or colored types. They contain large amounts of carbohydrates and proteins, but relatively little lipids. In general, they are widely consumed after boiling or used in desserts. In recent years, the polyphenols such as anthocyanins, catechins, and phenolic acids in beans, vegetables, and fruits have received much attention worldwide [1]. It is reported that common beans contain various polyphenols [2]. Phenolic compounds are synthesized by various enzymes in the biosynthetic pathways of common beans. For example, dihydro-flavonols can be converted to flavonols by flavonol synthase and to leucoanthocyanins by dihydroflavonol 4-reductase. Furthermore, leucoanthocyanins can be converted to anthocyanidins by anthocyanidin synthase or to flavonols by leucoanthocyanidin reductase. Phenolic glycosides are produced by glucosyltransferase. However, the key enzyme responsible for procyanidin condensation has not yet been identified $[3,4,5]$. Much has already been reported about the polyphenol content and the antioxidant and enzyme inhibition activity of common beans [6]. Nevertheless, the relative differences in their polyphenol composition and the correlations between their polyphenol compounds and antioxidant activity have seldom been addressed. In the present study, we analyzed the physicochemical characteristics of the polyphenols and antioxidants in common beans and identified the relation between seed coat color and total polyphenol content. We also characterized the traits of common beans known to have high antioxidant activity.

\section{Materials and Methods}

\subsection{Materials}

Twenty-eight common beans were harvested at Tokachi Agricultural Experiment Station, Hokkaido, Japan in 2014.

Folin-Ciocalteau reagent was purchased from Nacalai Tesque, Inc. (Kyoto, Japan). DPPH (2, 2-diphenyl-1picrylhydrazyl) was purchased from Tokyo Chemical Industry Co., Ltd. (Tokyo, Japan). Catechin, epicatechin, 
gallic acid, trolox, potassium ferricyanide and rutin were purchased from Sigma-Aldrich Co., LLC. (Tokyo, Japan). Cyanidin-3-O-glucoside and cyaniding-3-O-rutinoside were purchased from Extrasynthese Co. (Genay, France). Quercetin was purchased from Tokiwa Phytochemical Co., Ltd. (Tokyo, Japan). Ethanol, $\mathrm{Na}_{2} \mathrm{CO}_{3}, \mathrm{KH}_{2} \mathrm{PO}_{4}$, trichloroacetic acid, ferric chloride, vitamin $\mathrm{C}$ were purchased from Wako Pure Chemical Industries, Ltd. (Osaka, Japan). The other reagents were purchased from Funakoshi Co., Ltd. (Tokyo, Japan).

\subsection{Determination of Seed Coat Color}

Whole beans were transferred to petri dishes for 30 color determinations as defined by the International Commission on Illumination [7]. The Lab color space index was used to categorize seed color by lightness $\left(\mathrm{L}^{*}\right)$, redness $\left(a^{*}\right)$, and yellowness $\left(b^{*}\right)$. These measurements were made with a CR-400 colorimeter. Chroma ( $\left.\mathrm{C}^{*}\right)$ was calculated by redness and yellowness.

\subsection{Extraction and Determination of Total Polyphenols}

Polyphenols in the beans were extracted by the method of Saito [8]. Ground seeds (5 g) were placed in a falcon tube and mixed with $20 \mathrm{~mL}$ of $80 \% \mathrm{v} / \mathrm{v}$ ethanol, vortexed, and ultrasonicated for $30 \mathrm{~min}$. The suspension was then centrifuged at $1,006 \times g$ for $10 \mathrm{~min}$. The supernatant was transferred to another falcon tube to which $20 \mathrm{~mL}$ of $80 \%$ $\mathrm{v} / \mathrm{v}$ ethanol was added. Vortex-mixing, ultrasonication, and centrifugation were repeated twice. The final ethanol extract was mixed with $20 \mathrm{~mL}$ of $70 \% \mathrm{v} / \mathrm{v}$ acetone and the aforementioned process was repeated thrice to obtain an acetone extract. The total polyphenol content was determined by the Folin-Ciocalteau method [9] modified by using catechin as the standard at a concentration range of $0-0.25 \mathrm{mg} \mathrm{mL}^{-1}$. The extract $(100 \mu \mathrm{L})$ was placed in a micro-tube and mixed with $300 \mu \mathrm{L}$ distilled water, $400 \mu \mathrm{L}$ Folin-Ciocalteau reagent, and 400 of $10 \% \mathrm{Na}_{2} \mathrm{CO}_{3}$. The solution was then placed in a $30^{\circ} \mathrm{C}$ water bath for $30 \mathrm{~min}$. It was then centrifuged at $1,006 \times g$ for $10 \mathrm{~min}$ and its absorbance was read at $760 \mathrm{~nm}$. The results were expressed as mg catechin equivalents (CE) per gram seed $\left(y=10.953 x-0.0548, R^{2}=0.9965\right)$.

\subsection{Determination of Antioxidant Activity}

The DPPH radical scavenging activity was determined by the method of Brand-Williams [10]. The extract $(50 \mu \mathrm{L})$ was added to a microplate and mixed with $100 \mu \mathrm{L}$ of $99.5 \% \mathrm{v} / \mathrm{v}$ ethanol and $150 \mu \mathrm{L}$ DPPH solution. The solution was kept in the dark for 15 min after which its absorbance was determined at $520 \mathrm{~nm}$ by a microplate reader. Ten-fold diluted $2 \mathrm{mM}$ Trolox was used as the standard and the results were expressed as $\mu \mathrm{mol}$ Trolox equivalents (TE) per gram seed $(\mathrm{y}=-15.755 \mathrm{x}+26.858$, $\left.\mathrm{R}^{2}=0.9987\right)$.

The reducing power was determined by the method of Oyaizu [11]. The extract $(250 \mu \mathrm{L})$ was added to a microtube and mixed with $250 \mu \mathrm{L}$ of $\mathrm{KH}_{2} \mathrm{PO}_{4}$ buffer $(\mathrm{pH}$ $7.5)$ and $250 \mu \mathrm{L}$ of $1 \%(\mathrm{w} / \mathrm{v})$ potassium ferricyanide, then incubated at $50^{\circ} \mathrm{C}$ in a water bath for $20 \mathrm{~min}$. Then, 250 $\mu \mathrm{L}$ of $10 \%(\mathrm{w} / \mathrm{v})$ trichloroacetic acid was added and the mixture was centrifuged at $1,006 \times g$ for $10 \mathrm{~min}$. Then, 500 $\mu \mathrm{L}$ supernatant was transferred to another microtube and mixed with $500 \mu \mathrm{L}$ distilled water and $100 \mu \mathrm{L}$ of $0.1 \%$ $(\mathrm{w} / \mathrm{v})$ ferric chloride. After placing the solution in the dark for $15 \mathrm{~min}$, its absorbance was measured at $700 \mathrm{~nm}$. Vitamin C (1 mg mL $\left.\mathrm{m}^{-1}\right)$ was used as the standard and the results were expressed as $\mathrm{mg}$ vitamin $\mathrm{C}$ equivalents (VE) per gram seed $\left(y=8.7561 x+0.2194, R^{2}=0.9982\right)$.

\subsection{Identification of Polyphenols by RP-HPLC}

The polyphenol extracts were vacuum-dried and $20 \mu \mathrm{g}$ was re-dissolved in $200 \mu \mathrm{L}$ distilled water. The solution was filtered with a Minisart RC4 $(0.45 \mu \mathrm{m}$ pore diameter; Sartorius AG, Göttingen, Germany). The filtered sample $(20 \mu \mathrm{L})$ was injected into an RP-HPLC with a Shimadzu LC-6A HPLC system (LC-6A quaternary pump, SLC-6B system controller, CTO-6A column oven, SPD-10ADvp detector; Shimadzu Corporation, Kyoto, Japan), fitted with a Luna ${ }^{\circledR} \mathrm{RC} 18(2) 4.6 \times 250 \mathrm{~mm}, 5 \mu \mathrm{m}$ column (Phenomenex, Torrance, CA, USA). The temperature of the column oven was $40^{\circ} \mathrm{C}$ and the UV detection wavelength was $280 \mathrm{~nm}$. The initial column conditions were as follows: developing solvent A $(0.1 \% \mathrm{v} / \mathrm{v}$ trifluoroacetic acid) and eluent B $(0.1 \% \mathrm{v} / \mathrm{v}$ trifluoroacetic acid-acetonitrile). The gradient program followed was $0.01 \mathrm{~min}, 8 \% \mathrm{~B} ; 30 \mathrm{~min}, 30 \% \mathrm{~B} ; 40 \mathrm{~min}, 30 \% \mathrm{~B}$; $55 \mathrm{~min}$, $8 \% \mathrm{~B}$ at a flow rate of $1 \mathrm{~mL} \mathrm{~min}^{-1}$.

\subsection{Data Analysis}

The experiments were repeated at least three times. Data were expressed as means \pm standard deviation. Significant differences were determined by one-way ANOVA and Fisher's test (SAS v. 7.1, SAS Institute Inc., Cary, NC, USA). Differences were considered to be significant at $P<0.05$. Principal component analysis was performed with Ekuseru-Toukei v. 2008.

\section{Results and Discussion}

\subsection{Physical Properties of Common Beans}

In this study, we determined the weight and color of seeds of 28 common beans. The results are shown in Table 1. Lightness tended to decrease with increasing redness. There was a high positive correlation between total polyphenol content and redness $\left(\mathrm{a}^{*}\right)(\mathrm{r}=0.8753$; Figure 1). Similar correlations between polyphenol content and seed coat color are also reported for sweet potatoes and sorghum [12].

\subsection{Total Polyphenol and Antioxidant Activities in Common Beans}

The total polyphenol content and antioxidant activity of 28 common beans are shown in Table 2. In general, the polyphenol content in the white beans was significantly lower than that in colored beans. In this study, the red kidney bean had the highest polyphenol content $(6.12 \mathrm{mg} /$ 
g seed) and antioxidant activity $(21.98 \mu \mathrm{mol} / \mathrm{g}$ seed; 3.75 $\mathrm{mg} / \mathrm{g}$ seed). It is reported that white beans have no antioxidant activity, whereas it is the highest in red and black beans $[13,14]$. In another study, red kidney beans had the highest antioxidant activity, while white beans had the lowest [15]. In the present study, there were strong correlations between total polyphenol content and antioxidant activity $(\mathrm{r}=0.9484, \mathrm{r}=0.9815$ in Figure 2A and Figure2B). These results were similar to those reported for 29 common beans in the United States $(\mathrm{r}=$ $0.86, P<0.05)$ [16], and 15 fruits $\left(\mathrm{R}^{2}=0.99, \mathrm{R}>0.5\right)$ [17], and 6 cultivars of Iranian olive $\left(\mathrm{R}^{2}=0.976, P<0.05\right)[18]$. In this study, Taisho-Kintoki (red kidney) bean had the highest procyanidin content of all 28 common beans tested (5.7 mg CE / g seed). Nevertheless, it is still lower than that in chokeberry and grape [19,20].

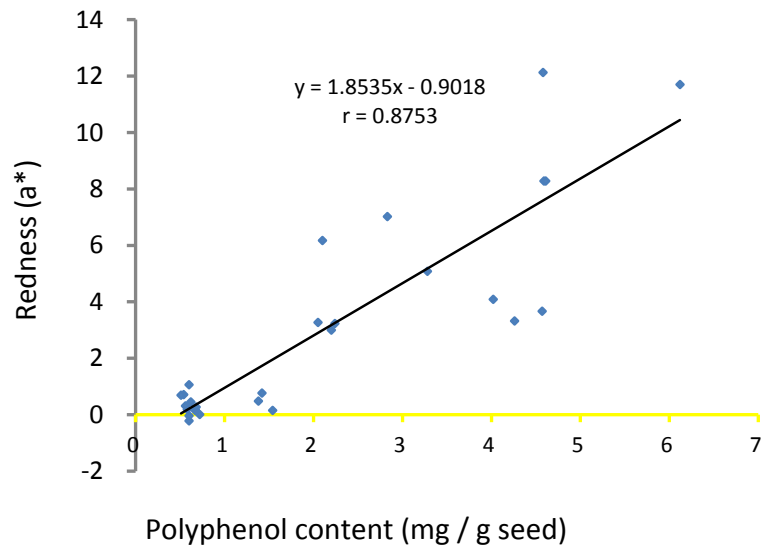

Figure 1. Correlation between polyphenol and redness ( $\left.a^{*}\right)$

Table 1. Physical Properties of 28 Common Beans

\begin{tabular}{|c|c|c|c|c|c|c|c|c|c|c|c|c|c|c|c|c|}
\hline \multirow{2}{*}{$\begin{array}{c}\text { No. } \\
1\end{array}$} & \multirow{2}{*}{$\begin{array}{l}\text { Sample } \\
\text { Yuki Tebou }\end{array}$} & \multirow{2}{*}{$\begin{array}{c}\text { Seed Color } \\
\text { White }\end{array}$} & \multicolumn{2}{|c|}{ Grain Weight (g) } & \multicolumn{3}{|c|}{ Lightness $\left(\mathrm{L}^{*}\right)$} & \multicolumn{3}{|c|}{ Redness $\left(a^{*}\right)$} & \multicolumn{3}{|c|}{ Yellowness $\left(b^{*}\right)$} & \multicolumn{3}{|c|}{ Chroma $\left(\mathrm{C}^{*}\right)$} \\
\hline & & & 0.29 & $\pm 0.021 \mathrm{~m}$ & 71.40 & \pm & $1.54 \mathrm{bc}$ & 0.71 & \pm & $0.24 \mathrm{ij}$ & 8.36 & \pm & $0.58 \mathrm{fg}$ & 8.40 & \pm & $0.58 \mathrm{~g}$ \\
\hline 2 & Gin Tebou & White & 0.31 & \pm 0.011 & 70.95 & \pm & $1.96 \mathrm{bc}$ & 0.19 & \pm & $0.22 \mathrm{klmn}$ & 7.62 & \pm & $0.45 \mathrm{hi}$ & 7.63 & \pm & $0.45 \mathrm{~h}$ \\
\hline 3 & Syo Tebou & White & 0.32 & $\pm 0.01 \mathrm{kl}$ & 69.65 & \pm & $0.90 \mathrm{c}$ & 0.69 & \pm & $0.16 \mathrm{ijk}$ & 8.38 & \pm & $0.20 \mathrm{fg}$ & 8.41 & \pm & $0.20 \mathrm{~g}$ \\
\hline 4 & Siro Nagauzura & White & 0.61 & $\pm 0.00 \mathrm{ef}$ & 55.22 & \pm & $2.80 \mathrm{fg}$ & 0.15 & \pm & $0.201 \mathrm{mn}$ & 6.58 & \pm & $0.46 \mathrm{kl}$ & 6.59 & \pm & $0.46 \mathrm{jk}$ \\
\hline 5 & Siro Maruuzura & White & 0.58 & $\pm 0.01 \mathrm{fg}$ & 51.93 & \pm & $2.26 \mathrm{hi}$ & 0.31 & \pm & $0.21 \mathrm{jklm}$ & 6.85 & \pm & $0.30 \mathrm{jk}$ & 6.86 & \pm & $0.30 \mathrm{ij}$ \\
\hline 6 & Fukusiro Kintoki & White & 0.85 & $\pm 0.08 \mathrm{ab}$ & 66.26 & \pm & $3.25 \mathrm{~d}$ & 0.17 & \pm & $0.301 \mathrm{mn}$ & 8.79 & \pm & $0.37 \mathrm{f}$ & 8.79 & \pm & $0.38 \mathrm{fg}$ \\
\hline 7 & Toya Daifuku & White & 0.87 & $\pm 0.02 \mathrm{a}$ & 73.68 & \pm & $4.32 \mathrm{a}$ & 0.22 & \pm & $0.18 n$ & 7.71 & \pm & $0.42 \mathrm{~h}$ & 7.72 & \pm & $0.42 \mathrm{~h}$ \\
\hline 8 & Kumamoto Ingen & White & 0.30 & $\pm 0.031 \mathrm{~m}$ & 72.51 & \pm & $2.07 \mathrm{ab}$ & 0.04 & \pm & $0.41 \mathrm{mn}$ & 13.70 & \pm & $1.08 \mathrm{a}$ & 13.70 & \pm & $1.08 \mathrm{bc}$ \\
\hline 9 & Navy Bean & White & 0.19 & $\pm 0.02 \mathrm{n}$ & 60.42 & \pm & $1.52 \mathrm{e}$ & 1.06 & \pm & $0.17 \mathrm{i}$ & 6.53 & \pm & $0.29 \mathrm{kl}$ & 6.62 & \pm & $0.28 \mathrm{jk}$ \\
\hline 10 & White Kidney & White & 0.54 & $\pm 0.05 \mathrm{gh}$ & 50.08 & \pm & $2.53 \mathrm{ij}$ & 0.45 & \pm & $0.17 \mathrm{jklm}$ & 5.00 & \pm & $0.38 \mathrm{mn}$ & 5.02 & \pm & $0.38 n$ \\
\hline 11 & Canellini & White & 0.55 & $\pm 0.01 \mathrm{gh}$ & 50.66 & \pm & $1.42 \mathrm{ij}$ & 0.27 & \pm & $0.10 \mathrm{jklmn}$ & 6.26 & \pm & 0.371 & 6.26 & \pm & $0.37 \mathrm{kl}$ \\
\hline 12 & Umakei No.5 & Half-spotted & 0.55 & $\pm 0.04 \mathrm{gf}$ & 69.86 & \pm & $2.84 \mathrm{c}$ & 0.01 & \pm & $0.251 \mathrm{mn}$ & 11.77 & \pm & $0.58 \mathrm{c}$ & 11.78 & \pm & $0.58 \mathrm{~d}$ \\
\hline 13 & Sihoro Ingen & Half-spotted & 0.66 & $\pm 0.02 \mathrm{~cd}$ & 55.23 & \pm & $3.29 \mathrm{fg}$ & 0.48 & \pm & $0.31 \mathrm{jkl}$ & 6.75 & \pm & $0.51 \mathrm{jkl}$ & 6.78 & \pm & 0.49ijk \\
\hline 14 & Taoca Bean & Half-spotted & 0.53 & $\pm 0.01 \mathrm{~h}$ & 53.77 & \pm & $7.23 \mathrm{gh}$ & 0.77 & \pm & $0.38 \mathrm{ij}$ & 7.14 & \pm & $1.68 \mathrm{ij}$ & 7.20 & \pm & $1.63 \mathrm{hi}$ \\
\hline 15 & Fuku Tora & Half-spotted & 0.81 & $\pm 0.04 \mathrm{~b}$ & 55.82 & \pm & $7.64 f$ & 3.23 & \pm & $2.37 \mathrm{gh}$ & 9.45 & \pm & $2.28 \mathrm{e}$ & 10.13 & \pm & $2.80 \mathrm{e}$ \\
\hline 16 & Beni Sibori & Half-spotted & 0.63 & $\pm 0.04 \mathrm{cde}$ & 41.93 & \pm & $5.57 \mathrm{k}$ & 3.00 & \pm & $1.08 \mathrm{~h}$ & 4.77 & \pm & 1.94 no & 5.88 & \pm & 1.421 \\
\hline 17 & Hakusai Bean & Half-spotted & 0.42 & $\pm 0.02 \mathrm{j}$ & 38.06 & \pm & $2.211 \mathrm{~m}$ & 4.09 & \pm & $0.72 \mathrm{f}$ & 5.51 & \pm & $0.84 \mathrm{~m}$ & 6.93 & \pm & $0.52 \mathrm{ij}$ \\
\hline 18 & Siroji Biruma & Spotted & 0.26 & $\pm 0.01 \mathrm{~m}$ & 49.10 & \pm & $2.32 \mathrm{j}$ & 6.17 & \pm & $0.54 \mathrm{~d}$ & 12.85 & \pm & $0.64 b$ & 14.26 & \pm & $0.80 \mathrm{ab}$ \\
\hline 19 & Shell Bean & Spotted & 0.62 & $\pm 0.04 \mathrm{def}$ & 35.05 & \pm & $1.85 \mathrm{no}$ & 3.27 & \pm & $1.07 \mathrm{gh}$ & 4.11 & \pm & $1.62 \mathrm{pq}$ & 5.26 & \pm & $1.92 \mathrm{mn}$ \\
\hline 20 & Futsu Biruma & Spotted & 0.32 & $\pm 0.02 \mathrm{kl}$ & 35.95 & \pm & $1.49 n$ & 7.02 & \pm & $1.06 \mathrm{c}$ & 6.76 & \pm & $1.16 \mathrm{jkl}$ & 9.77 & \pm & $1.43 \mathrm{e}$ \\
\hline 21 & Kiji Bean & Spotted & 0.52 & $\pm 0.03 \mathrm{~h}$ & 39.14 & \pm & 2.281 & 12.13 & \pm & $0.76 \mathrm{a}$ & 8.24 & \pm & $1.27 \mathrm{~g}$ & 14.71 & \pm & $0.85 \mathrm{a}$ \\
\hline 22 & Triumph De Francy & Spotted & 0.33 & $\pm 0.01 \mathrm{kl}$ & 35.31 & \pm & 1.81 no & 3.67 & \pm & $0.93 \mathrm{fg}$ & 4.35 & \pm & $1.52 \mathrm{op}$ & 5.69 & \pm & $1.751 \mathrm{~m}$ \\
\hline 23 & Karasu Ingen & Black & 0.36 & $\pm 0.01 \mathrm{k}$ & 33.42 & \pm & 0.680 & 0.15 & \pm & $0.081 \mathrm{mn}$ & -0.12 & \pm & $0.11 \mathrm{~s}$ & 0.22 & \pm & 0.08 o \\
\hline 24 & Gokuwase Murasaki Saya & Brown & 0.46 & $\pm 0.01 \mathrm{i}$ & 59.63 & \pm & $1.63 \mathrm{e}$ & 5.08 & \pm & $0.33 \mathrm{e}$ & 14.15 & \pm & $0.47 \mathrm{a}$ & 15.04 & \pm & $0.51 \mathrm{a}$ \\
\hline 25 & Sutton's Premier & Brown & 0.62 & $\pm 0.02 \mathrm{ef}$ & 42.16 & \pm & $1.52 \mathrm{k}$ & 3.32 & \pm & $0.58 \mathrm{gh}$ & 9.64 & \pm & $0.85 \mathrm{e}$ & 10.21 & \pm & $0.85 \mathrm{e}$ \\
\hline 26 & Jaula & Brown & 0.55 & $\pm 0.01 \mathrm{gh}$ & 42.68 & \pm & $0.82 \mathrm{k}$ & 8.28 & \pm & $0.37 b$ & 10.39 & \pm & $0.48 \mathrm{~d}$ & 13.29 & \pm & $0.48 \mathrm{c}$ \\
\hline 27 & Roxo & Red & 0.25 & $\pm 0.00 \mathrm{~m}$ & 38.14 & \pm & $0.731 \mathrm{~m}$ & 8.28 & \pm & $0.26 \mathrm{~b}$ & 3.76 & \pm & $0.25 q$ & 9.10 & \pm & $0.27 f$ \\
\hline 28 & Taisho Kintoki & Red & 0.66 & $\pm 0.01 \mathrm{c}$ & 36.56 & \pm & $1.13 \mathrm{mn}$ & 11.70 & \pm & $0.68 \mathrm{a}$ & 2.87 & \pm & $0.23 \mathrm{r}$ & 12.05 & \pm & $0.70 \mathrm{~d}$ \\
\hline
\end{tabular}

Data are means \pm SD from at least three independent studies. Values with different letters within the same column are significantly different at $P<0.05$.

A

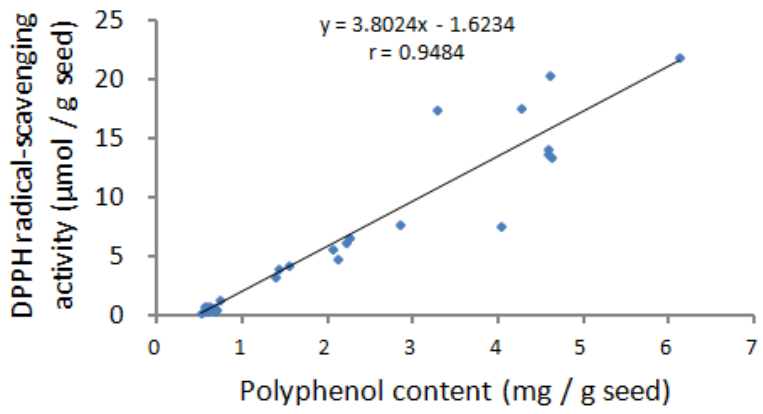

$\mathrm{B}$

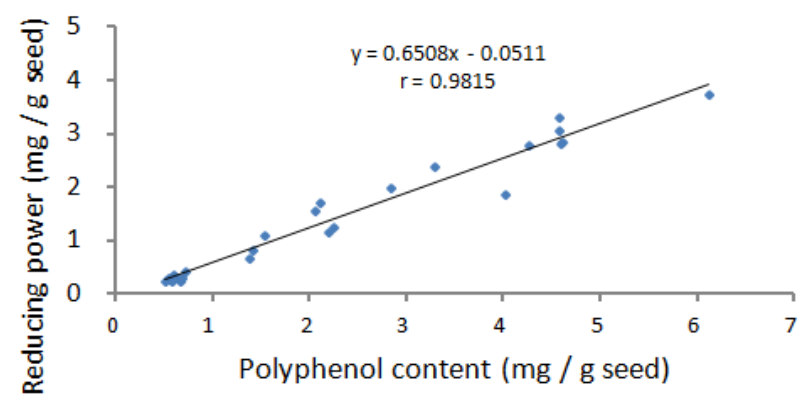

Figure 2. Correlation between polyphenol and antioxidant activity. A. Correlation between polyphenol and DPPH radical-scavenging activity. B. Correlation between polyphenol and reducing power 
Table 2. Polyphenol and Antioxidant Activities of 28 Common Beans

\begin{tabular}{|c|c|c|c|c|c|c|c|c|c|c|c|}
\hline \multirow{2}{*}{$\frac{\text { No. }}{1}$} & \multirow{2}{*}{$\frac{\text { Sample }}{\text { Yuki Tebou }}$} & \multirow{2}{*}{$\frac{\text { Seed Color }}{\text { White }}$} & \multicolumn{3}{|c|}{$\begin{array}{l}\text { Polyphenol } \\
\text { (mg/g seed) }\end{array}$} & \multicolumn{3}{|c|}{$\begin{array}{l}\text { DPPH radical-scavenging activity } \\
(\mu \mathrm{mol} / \mathrm{g} \text { seed })\end{array}$} & \multicolumn{3}{|c|}{$\begin{array}{l}\text { Reducing power } \\
\text { (mg / g seed) }\end{array}$} \\
\hline & & & 0.54 & \pm & $0.01 \mathrm{t}$ & 0.83 & \pm & $0.04 \mathrm{~s}$ & 0.31 & \pm & $0.01 \mathrm{rst}$ \\
\hline 2 & Gin Tebou & White & 0.58 & \pm & $0.01 \mathrm{~s}$ & 0.44 & \pm & $0.02 \mathrm{w}$ & 0.26 & \pm & $0.01 \mathrm{u}$ \\
\hline 3 & Syo Tebou & White & 0.51 & \pm & $0.01 \mathrm{u}$ & 0.28 & \pm & $0.07 x$ & 0.26 & \pm & $0.01 \mathrm{u}$ \\
\hline 4 & Siro Nagauzura & White & 0.67 & \pm & $0.01 \mathrm{p}$ & 0.53 & \pm & $0.05 \mathrm{vw}$ & 0.26 & \pm & $0.01 \mathrm{u}$ \\
\hline 5 & Siro Maruuzura & White & 0.56 & \pm & $0.01 \mathrm{t}$ & 0.88 & \pm & $0.03 \mathrm{rs}$ & 0.29 & \pm & 0.01 tu \\
\hline 6 & Fukusiro Kintoki & White & 0.67 & \pm & $0.01 \mathrm{p}$ & 0.73 & \pm & $0.05 \mathrm{t}$ & 0.29 & \pm & $0.01 \mathrm{stu}$ \\
\hline 7 & Toya Daifuku & White & 0.60 & \pm & $0.01 \mathrm{rs}$ & 0.63 & \pm & $0.08 \mathrm{u}$ & 0.33 & \pm & $0.01 \mathrm{r}$ \\
\hline 8 & Kumamoto Ingen & White & 0.60 & \pm & $0.01 \mathrm{rs}$ & 0.78 & \pm & $0.02 \mathrm{st}$ & 0.34 & \pm & $0.01 \mathrm{qr}$ \\
\hline 9 & Navy Bean & White & 0.60 & \pm & $0.01 \mathrm{qr}$ & 0.96 & \pm & $0.06 \mathrm{r}$ & 0.37 & \pm & $0.01 \mathrm{q}$ \\
\hline 10 & White Kidney & White & 0.62 & \pm & $0.01 \mathrm{q}$ & 0.81 & \pm & $0.04 \mathrm{st}$ & 0.33 & \pm & $0.01 \mathrm{rs}$ \\
\hline 11 & Canellini & White & 0.68 & \pm & $0.01 \mathrm{p}$ & 0.58 & \pm & 0.04uv & 0.33 & \pm & $0.01 \mathrm{r}$ \\
\hline 12 & Umakei No.5 & Half-spotted & 0.72 & \pm & 0.010 & 1.38 & \pm & $0.07 \mathrm{q}$ & 0.43 & \pm & $0.01 \mathrm{p}$ \\
\hline 13 & Sihoro Ingen & Half-spotted & 1.38 & \pm & $0.02 \mathrm{n}$ & 3.43 & \pm & $0.03 p$ & 0.69 & \pm & 0.010 \\
\hline 14 & Taoca Bean & Half-spotted & 1.42 & \pm & $0.01 \mathrm{~m}$ & 4.03 & \pm & $0.03 \mathrm{o}$ & 0.83 & \pm & $0.01 \mathrm{n}$ \\
\hline 15 & Fuku Tora & Half-spotted & 2.24 & \pm & $0.01 \mathrm{~h}$ & 6.70 & \pm & $0.04 \mathrm{j}$ & 1.26 & \pm & $0.01 \mathrm{k}$ \\
\hline 16 & Beni Sibori & Half-spotted & 2.20 & \pm & $0.02 \mathrm{i}$ & 6.32 & \pm & $0.05 \mathrm{k}$ & 1.17 & \pm & 0.011 \\
\hline 17 & Hakusai Bean & Half-spotted & 4.02 & \pm & $0.02 \mathrm{e}$ & 7.74 & \pm & $0.04 \mathrm{i}$ & 1.87 & \pm & $0.01 \mathrm{~h}$ \\
\hline 18 & Siroji Biruma & Spotted & 2.10 & \pm & $0.02 \mathrm{j}$ & 4.96 & \pm & $0.04 \mathrm{~m}$ & 1.72 & \pm & $0.04 \mathrm{i}$ \\
\hline 19 & Shell Bean & Spotted & 2.05 & \pm & $0.02 \mathrm{k}$ & 5.79 & \pm & 0.081 & 1.57 & \pm & $0.04 \mathrm{j}$ \\
\hline 20 & Futsu Biruma & Spotted & 2.83 & \pm & $0.02 \mathrm{~g}$ & 7.85 & \pm & $0.04 \mathrm{~h}$ & 2.01 & \pm & $0.02 \mathrm{~g}$ \\
\hline 21 & Kiji Bean & Spotted & 4.58 & \pm & $0.02 b c$ & 13.80 & \pm & $0.05 f$ & 3.08 & \pm & $0.05 \mathrm{c}$ \\
\hline 22 & Triumph De Francy & Spotted & 4.57 & \pm & $0.01 \mathrm{c}$ & 14.27 & \pm & $0.01 \mathrm{e}$ & 3.33 & \pm & $0.06 \mathrm{~b}$ \\
\hline 23 & Karasu Ingen & Black & 1.54 & \pm & 0.021 & 4.43 & \pm & $0.03 n$ & 1.12 & \pm & $0.02 \mathrm{~m}$ \\
\hline 24 & Gokuwase Murasaki Saya & Brown & 3.28 & \pm & $0.13 \mathrm{f}$ & 17.53 & \pm & $0.22 \mathrm{~d}$ & 2.39 & \pm & $0.01 \mathrm{f}$ \\
\hline 25 & Sutton's Premier & Brown & 4.26 & \pm & $0.07 \mathrm{~d}$ & 17.67 & \pm & $0.06 \mathrm{c}$ & 2.79 & \pm & $0.02 \mathrm{e}$ \\
\hline 26 & Jaula & Brown & 4.59 & \pm & $0.08 b c$ & 20.51 & \pm & $0.04 \mathrm{~b}$ & 2.82 & \pm & $0.06 \mathrm{e}$ \\
\hline 27 & Roxo & Red & 4.61 & \pm & $0.11 \mathrm{~b}$ & 13.58 & \pm & $0.32 \mathrm{~g}$ & 2.86 & \pm & $0.04 \mathrm{~d}$ \\
\hline 28 & Taisho Kintoki & Red & 6.12 & \pm & $0.19 \mathrm{a}$ & 21.98 & \pm & $0.30 \mathrm{a}$ & 3.75 & \pm & $0.05 \mathrm{a}$ \\
\hline
\end{tabular}

Data are means \pm SD from at least three independent studies. Values with different letters within the same column are significantly different at $P<0.05$.

Table 3. Polyphenols Compositions of 28 Common Beans

\begin{tabular}{|c|c|c|c|c|c|c|c|c|c|c|c|c|c|c|c|c|}
\hline Peak & Compound & $\mathrm{tR} \min$ & YuT & GiT & SyT & $\mathrm{SiN}$ & SiM & FuK & ToD & $\mathrm{KuI}$ & $\mathrm{NaB}$ & WKB & Cal & SiI & $\mathrm{UmN}$ & $\mathrm{TaB}$ \\
\hline 1 & Gallic acid & 5.29 & 1.6 & 0.6 & 0.6 & 0.8 & 1.1 & 0.7 & 0.8 & 1.1 & 1.0 & 0.7 & 0.8 & 0.5 & 1.1 & 1.4 \\
\hline 2 & Catechin-7-O-glucoside & 6.17 & 8.8 & 14.1 & 8.0 & 9.0 & 9.4 & 16.1 & 17.0 & 7.3 & 9.7 & 9.7 & 14.4 & 9.7 & 6.2 & 8.7 \\
\hline 3 & Procyanidin dimer & 8.56 & 4.1 & 2.5 & 2.7 & 4.5 & 5.1 & 4.4 & 3.2 & 3.8 & 4.4 & 4.5 & 4.7 & 4.7 & 4.2 & 3.0 \\
\hline 4 & Catechin & 9.23 & 7.6 & 5.5 & 5.9 & 10.4 & 9.2 & 8.8 & 7.5 & 8.9 & 8.5 & 9.7 & 8.7 & 7.7 & 8.2 & 5.2 \\
\hline 5 & 3-caffeoylquinic acid & 10.24 & 3.4 & 1.9 & 1.7 & 2.1 & 2.3 & 2.1 & 1.9 & 2.1 & 2.3 & 2.0 & 1.6 & 3.2 & 1.9 & 2.4 \\
\hline 6 & 5-caffeoylquinic acid & 11.39 & 5.3 & 3.5 & 3.9 & 3.8 & 2.7 & 3.3 & 3.5 & 3.0 & 3.1 & 3.9 & 3.0 & 3.3 & 3.6 & 2.4 \\
\hline 7 & Epicatechin & 12.47 & 3.6 & 2.7 & 2.6 & 2.2 & 2.4 & 1.9 & 3.1 & 2.8 & 2.7 & 2.1 & 2.1 & 2.5 & 2.7 & 2.6 \\
\hline 8 & Cyanidin-3-O-glucoside & 14.36 & - & - & - & - & 0.4 & - & - & 0.7 & 0.5 & 0.5 & 0.6 & 0.4 & 0.5 & 1.1 \\
\hline 9 & Cyanidin-3-O-rutinoside & 15.29 & - & - & 0.5 & 0.6 & 0.5 & 0.6 & 0.8 & 0.6 & 0.5 & 0.8 & 0.6 & 0.9 & 0.9 & 0.7 \\
\hline 10 & Rutin & 20.25 & - & - & - & - & - & - & - & - & - & - & - & 0.8 & - & 0.6 \\
\hline 11 & Kaempferol-3-O-glucoside & 22.99 & - & - & - & - & - & - & - & - & - & - & - & 0.5 & - & 0.9 \\
\hline 12 & Quercetin & 24.94 & - & - & 0.5 & - & - & - & - & - & - & - & - & - & - & - \\
\hline 13 & Others & - & 65.6 & 69.3 & 73.4 & 66.6 & 67.0 & 62.1 & 62.3 & 69.8 & 67.3 & 66.1 & 63.5 & 65.9 & 70.8 & 71.2 \\
\hline
\end{tabular}

\begin{tabular}{|c|c|c|c|c|c|c|c|c|c|c|c|c|c|c|c|}
\hline Peak & Compound & FuT & $\mathrm{BeS}$ & FuB & $\mathrm{SiB}$ & $\mathrm{KiB}$ & TDF & ShB & GMS & $\mathrm{TaK}$ & $\mathrm{HaB}$ & $\mathrm{CaI}$ & Jal & $\mathrm{SuP}$ & Rox \\
\hline 1 & Gallic acid & 0.6 & 1.9 & 1.0 & 0.7 & 1.4 & 1.4 & 1.7 & 4.3 & 3.5 & 2.5 & 0.6 & 2.7 & 3.4 & 1.7 \\
\hline 2 & Catechin-7-O-glucoside & 7.6 & 6.2 & 2.0 & 2.0 & 1.6 & 2.6 & 2.8 & - & - & 3.7 & - & - & - & - \\
\hline 3 & Procyanidin dimer & 5.0 & 4.3 & 9.2 & 6.3 & 3.0 & 3.1 & 6.6 & 15.5 & 7.0 & 3.6 & 1.9 & 3.5 & 2.0 & 1.3 \\
\hline 4 & Catechin & 7.4 & 5.8 & 3.2 & 3.7 & 3.4 & 3.0 & 3.5 & 16.7 & 10.5 & 3.2 & 6.0 & 2.8 & 1.3 & 2.5 \\
\hline 5 & 3-caffeoylquinic acid & 3.3 & 3.0 & 2.4 & 2.1 & 1.6 & 1.2 & 3.0 & 2.7 & 3.6 & 2.0 & 1.7 & 0.6 & 0.7 & 0.8 \\
\hline 6 & 5-caffeoylquinic acid & 2.8 & 2.9 & 1.9 & 1.9 & 2.4 & 2.2 & 3.0 & 2.3 & 2.7 & 4.0 & 4.2 & 4.2 & 3.4 & 4.9 \\
\hline 7 & Epicatechin & 3.8 & 2.2 & 2.2 & 2.3 & 0.9 & 2.2 & 3.0 & 4.5 & 2.9 & 1.6 & 3.1 & 0.7 & 0.6 & 2.0 \\
\hline 8 & Cyanidin-3-O-glucoside & 1.4 & 3.2 & 1.4 & 1.8 & 1.1 & 1.4 & 1.3 & 3.9 & 3.1 & 1.1 & 2.4 & - & 0.5 & 1.7 \\
\hline 9 & Cyanidin-3-O-rutinoside & 1.2 & 0.9 & 1.0 & 1.1 & 0.7 & - & 0.8 & 2.0 & 9.3 & 0.5 & 1.0 & - & 0.4 & 2.7 \\
\hline 10 & Rutin & - & 0.5 & - & - & - & - & 1.5 & 0.9 & - & 1.7 & 8.5 & 1.0 & 1.0 & 3.0 \\
\hline 11 & Kaempferol-3-O-glucoside & - & - & - & - & 1.4 & 1.1 & 1.1 & 1.8 & 4.2 & 36.9 & 31.5 & 72.6 & 70.0 & 51.2 \\
\hline 12 & Quercetin & - & - & - & - & - & - & 1.1 & - & - & 4.5 & 1.4 & 3.1 & 3.3 & 4.4 \\
\hline 13 & Others & 67.0 & 69.2 & 75.8 & 78.1 & 82.4 & 81.8 & 70.8 & 45.5 & 53.3 & 34.7 & 37.7 & 8.7 & 13.3 & 23.9 \\
\hline
\end{tabular}

Data are expressed as \% of total area and represent the relative abundance of a compound relative to the sum of areas of all peaks in the chromatogram for each cultivar. Identification was based on pure standards. YuT: Yuki-Tebou; GiT: Gin-Tebou; SyT: Syo-Tebou; SiN: Siro-Nagauzura; SiM: SiroMaruuzura; FuK: Fukusiro-Kintoki; ToD: Toya-Daifuku; KuI: Kumamoto-Ingen; NaB: Navy-Bean; WKB: White Kidney Bean; Cal: Canellini; SiI: Sihoro-Ingen; UmN: Umakei-No.5; TaB: Taoca-Bean; FuT: Fuku-Tora; BeS: Beni-Sibori; FuB: Futsu-Biruma; SiB: Siroji-Biruma KiB: Kiji-Bean; TDF: Triumph De Francy; ShB: Shell Bean; GMS: Gokuwase-Murasaki-Saya; TaK: Taisho-Kintoki; HaB: Hakusai-Bean; CaI: Carasu-Ingen; Jal: Jaula; SuP: Sutton's Premier; Rox: Roxo. 


\subsection{Polyphenol Compounds in Common Beans}

A total of 12 phenolic compounds were identified in the 28 common beans (Table 3 ). The relative abundance of a compound is expressed as the ratio of the sum of areas of all peaks in the chromatogram for each cultivar. Gallic acid (0.50-4.26\%), procyanidin dimer (1.31-15.47\%), catechin (1.34-16.72\%), 3-caffeoylquinic acid (0.64-3.55\%), 5-caffeoylquinic acid (1.88-5.34\%), and epicatechin (0.63$4.50 \%$ ) were found in all samples and have been previously identified in common beans [21]. Catechin-7O-glucoside (1.55-16.96\%) was found in 22 samples. It was observed that white colored common beans tend to have relatively high levels of catechin-7-O-glucoside. Cyanidin-3-O-glucoside (0.41-3.89\%) was found in 21 cultivars and cyanidin-3-O-rutinoside (0.40-9.34\%) was found 24 samples. Colored common beans have high relative abundances of anthocyanins. These compounds have been reported elsewhere for common beans [22]. Rutin (0.51-8.51\%), quercetin (0.52-4.48\%), and kaempferol-3-O-glucoside $(0.48-72.63 \%)$ were identified in ten, seven, and twelve common beans, respectively. Quercetin and kaempferol were identified in 10 common beans at levels of 14.1-24.2 $\mu \mathrm{g} \mathrm{g}^{-1}$ and 11.4-61.0 $\mu \mathrm{g} \mathrm{g}^{-1}$, respectively [23].

Principal component analysis of 12 identified compounds and antioxidant activity of 28 common beans indicated that the first two principal components had eigenvalues explaining $64.8 \%$ of the total variance $(\mathrm{PC} 1=$ 38.0; PC2 = 26.8) (Figure 3). In this study, 28 common beans were divided into three groups according to their phenolic compound content. Group one contained 14 common beans like white beans and several half-spotted beans. This cluster had positive scores in PC2 and negative scores in PC1. They were positively correlated with caffeoylquinic acid and catechin-7-O-glucoside and negatively correlated with antioxidant activity. Similarly, the cluster containing nine common beans like TaishoKintoki and Gokuwase-Murasaki-Saya presented higher values than group 1 based on $\mathrm{PC} 1$, and large negative scores in PC2. This group correlated with anthocyanin and procyanidin dimer and the samples had higher antioxidant activity than samples of group 1. Cluster 3 contained five samples like Jaula and Sutton's Premier. It had large positive scores in PC1. They were positively correlated with kaempferol-3-O-glucoside, quercetin, and antioxidant activity. The main polyphenol compounds detected in common beans were phenolic acid, flavonol, anthocyanin, and tannin. Their functions include antioxidant activity and disease resistance. It was reported that kaempferol glycosides are hepatoprotectant and inhibit $\alpha$-glucosidase $[24,25]$. Anthocyanins and proanthocyanidins from adzuki bean were found to have strong antioxidant activity $[26,27]$. Common beans with high antioxidant activity may contain large amounts of kaempferol-3-O-glucoside, which is synthesized by key enzymes flavonol synthase and glycosyltransferase or substantial quantities of cyanidin glycosides synthesized by dihydroflavonol 4-reductase, anthocyanidin synthase, and glycosyltransferase. The content of these enzymes in common beans may determine the strength of their antioxidant activity. In future research, this information could be used to breed new cultivars of common beans with high antioxidant activity.

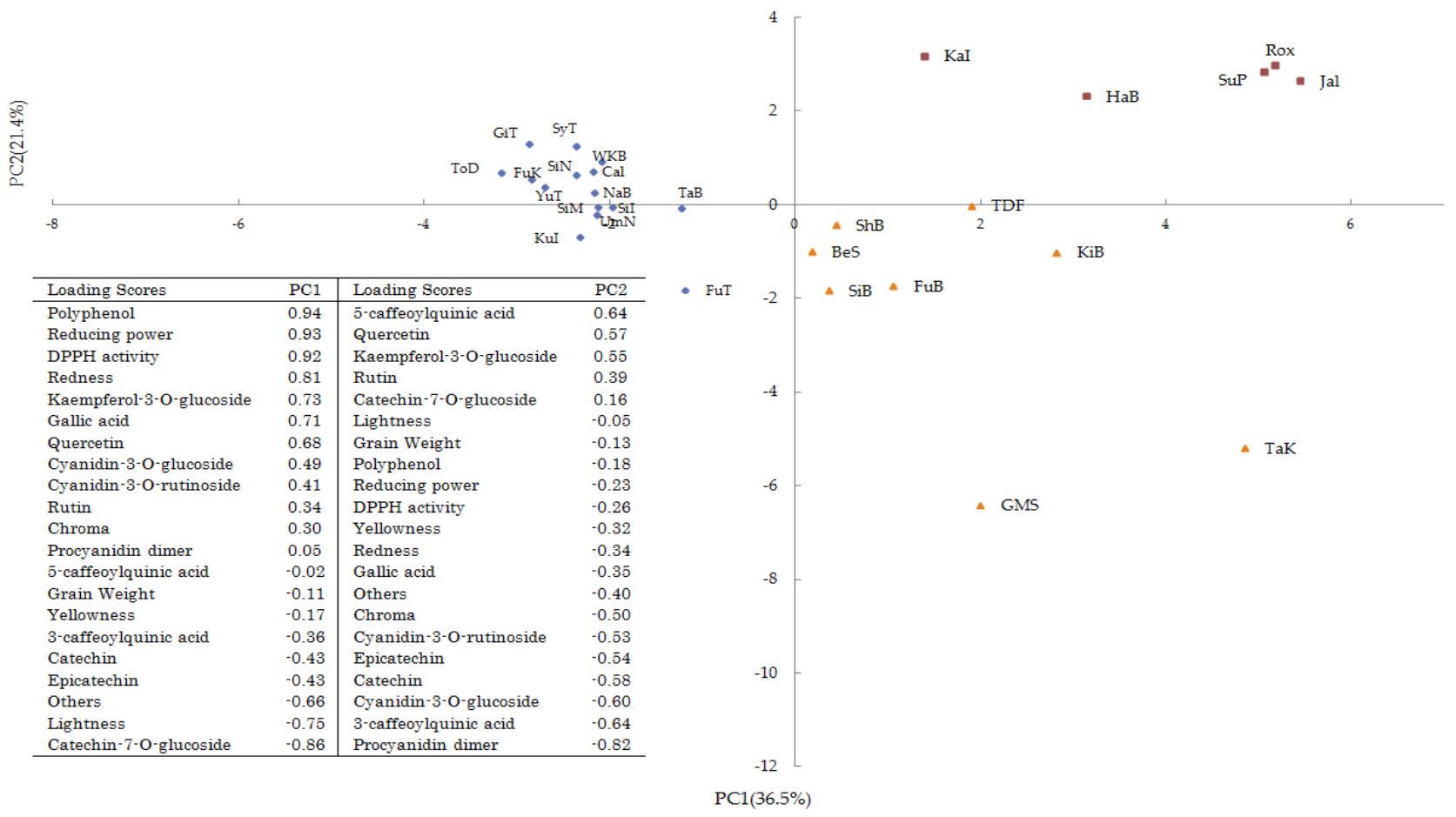

Figure 3. Cluster and principal component analysis score plot of 28 common beans. Score plots for the first two principal components, PC1 (36.5) and PC2 (21.4). Results are expressed as \% of total area and represent the relative abundance of a compound relative to the sum of areas of all peaks in the chromatogram for each cultivar. Identification was based on pure standards. YuT: Yuki-Tebou; GiT: Gin-Tebou; SyT: Syo-Tebou; SiN: Siro-Nagauzura; SiM: Siro-Maruuzura; FuK: Fukusiro-Kintoki; ToD: Toya-Daifuku; KuI: Kumamoto-Ingen; NaB: Navy-Bean; WKB: White Kidney Bean; Cal: Canellini; SiI: Sihoro-Ingen; UmN: Umakei-No.5; TaB: Taoca-Bean; FuT: Fuku-Tora; BeS: Beni-Sibori; FuB: Futsu-Biruma; SiB: Siroji-Biruma KiB: Kiji-Bean; TDF: Triumph De Francy; ShB: Shell Bean; GMS: Gokuwase-Murasaki-Saya; TaK: Taisho-Kintoki; HaB: Hakusai-Bean; CaI: CarasuIngen; Jal: Jaula; SuP: Sutton's Premier; Rox: Roxo 


\section{Conclusion}

There is a strong correlation between total polyphenol content and redness $\left(\mathrm{a}^{*}\right)$ in common beans. Antioxidant activity in colored beans is higher than it is in white beans. There is a high positive correlation between the total polyphenol content and antioxidant activity. In general, common beans are abundant in polyphenols. Common beans with high antioxidant activity not only have high total polyphenol content, but also elevated levels of particular polyphenols. The biosynthetic enzymes flavonol synthase, dihydroflavonol 4-reductase, and anthocyanidin synthase are positively correlated with antioxidant activity in common beans.

\section{Acknowledgments}

The authors thank Prof. X. Q. Jiang of the Department of Agricultural Economics, Graduate School of Animal Husbandry, Obihiro University of Agriculture and Veterinary Medicine, Obihiro, Hokkaido, Japan.

\section{Statement of Competing Interests}

The authors have no competing interests.

\section{List of Abbreviations}

CE: catechin equivalents; DPPH: 2, 2-diphenyl-1picrylhydrazyl; TE: Trolox equivalents; VE: vitamin $\mathrm{C}$ equivalents; PC: principal component

\section{References}

[1] Osegi, K., Yamada, C., and Izumi, H., "Functionality of polyphenols," Nagoya Nutrition Science Magazine, 1. 1-7. 2015.

[2] Takahata, Y., Ohnishi-Kameyama, M., Furuta, S., Takahashi, M., and Suda, I., "Highly polymerized procyanidins in brown soybean seed coat with a high radical-scavenging activity," Journal of Agricultural and Food Chemistry, 49(12). 5843-5847. 2001.

[3] Winkel-Shirley, B., "Flavonoid biosynthesis, A colorful model for genetics, biochemistry, cell biology, and biotechnology," Plant Physiology, 126(2). 485-493. 2001.

[4] Saijo, R., "Metabolic maps of flavonoids," Tea Research Journal, 11. 79-88. 2012.

[5] He, F., Pan, Q.H., Shi, Y., and Duan, C.Q., "Biosynthesis and genetic regulation of proanthocyanidins in plants," Molecules, 13(10). 2674-2703. 2008.

[6] Barrett, M.L., and Udani, J.K., “A proprietary $\alpha$-amylase inhibitor from white bean (Phaseolus vulgaris): a review of clinical studies on weight loss and glycemic control," Nutrition Journal, 10. 24. 2011.

[7] CIE, Colorimetry (Second Edition), Central Bureau of the Commission International de L'Eclairage, Vienna, 1986.

[8] Saito, Y., Nishi, S., Koaze, H., Hironaka, K., Kojima, M., "Antioxidant and inhibitory activity on $\alpha$-amylase and $\alpha$ glucosidase in legume polyphenols," Journal of the Japanese Society for Food Science and Technology, 53(12). 563-567. 2007.

[9] Saito, Y., "Characterization and biosynthesis regulation of polyphenol in legumes seed," Journal of the Japanese Society for Food Science and Technology, 53(7). 380-385. 2010.
[10] Brand-Williams, W., Cuvelier, M.E., and Berset, C. "Use of a free radical method to evaluate antioxidant activity," Food Science and Technology, 28(1). 25-30. 1995.

[11] Oyaizu, M., "Studies on products of browning reaction. (Antioxidative activities of products of browning reaction prepared from glucosamine)," The Japanese Journal of Nutrition and Dietetics, 44(6). 307-315. 1986.

[12] Awika. J.M., Rooney, L.W., Wu, X., Prior, R.L., and CisnerosZevallos, L., "Screening methods to measure antioxidant activity of sorghum (Sorghum bicolor) and sorghum products," Journal of Agricultural and Food Chemistry, 51(23). 6657-6662. 2003.

[13] Laparra, J.M., Glahn, R.P, and Miller, D.D., "Bioaccessibility of phenols in common beans (Phaseolus vulgaris L.) and iron (Fe) availability to Caco-2 cells," Journal of Agricultural and Food Chemistry, 56(22). 10999-11005. 2008.

[14] Tsuda, T., Watanabe, M., Ohshima, K., Norinobu, S., Choi, S., Kawakishi, S., and Osawa, T., "Anti-oxidative activity of the anthocyanin pigments cyanidin3-O- $\beta$-D-glucoside and cyanidin," Journal of Agricultural and Food Chemistry, 42(11). 2407-2410. 1994.

[15] Oomah, B.D., Cardador-Martinez, A., and Loarca-Pina, G., "Phenolics and anti-oxidative activities in common beans (Phaseolus vulgaris L.)," Journal of the Science of Food and Agriculture, 85. 935-942. 2005.

[16] Akond, ASMGM., Khandaker, L., Berthold, J., Gates, L., Peters, K., Delong, H., and Hossain, K., "Anthocyanin, total polyphenols and antioxidant activity of common bean," American Journal of Food Technology, 6(5). 385-394. 2011.

[17] Miyashita, J., and Kojima, M., "The correlation between polyphenol quantity and anti-oxidative property which are included in the plum and the blueberry," Academic Research Report of Obihiro University of Agriculture and Veterinary Medicine, 26. 13-19. 2005.

[18] Haji-mahmoodi, M., Sadeghi, N., Jannat, B., Oveisi, M.R., Madani, S., Kiayi, M., Akrami, M.R., and Ranjbar, A.M., "Antioxidant activity, reducing power and total phenolic content of Iranian olive cultivar," Journal of Biological Sciences, 8(4). 779-783. 2008.

[19] Kulling, S.E., and Rawel, H.M., "Chokeberry (Aronia melanocarpa) - A review on the characteristic components and potential health effects," Planta Medica, 74(13). 1625-1634. 2008.

[20] Quiñones, M., Guerrero, L., Suarez, M., Pons, Z., Aleixandre, A., Arola, L., and Muguerz, B., "Low-molecular procyanidin-rich grape seed extract exerts antihypertensive effect in males spontaneously hypertensive rats," Food Research International, 51. 587-595. 2013.

[21] Luis, M., Allison, M., Mark, A.B., and Elvira, G., "Bean cultivars (Phaseolus vulgaris L.) have similar high antioxidant capacity, in vitro inhibition of $\alpha$-amylase and $\alpha$-glucosidase while diverse phenolic composition and concentration," Food Research International, 69. 38-48. 2015.

[22] Aparicio-Fernandez, X., Manzo-Bonilla, L., and Loarca-Pina, G., "Comparison of antimutagenic activity of phenolic compounds in newly harvested and stored common beans Phaseolus vulgaris against Aflatoxin $\mathrm{B}_{1}$," Journal of Food Science, 70. 73-78. 2005.

[23] Doria, E., Campion, B., Sparvoli, F., Tava, A., and Nielesen, E., "Anti-nutrient components and metabolites with health implications in seeds of 10 common bean (Phaseolus vulgaris L. and Phaseolus lunatus L.) landraces cultivated in southern Italy," Journal of Food Composition and Analysis, 26. 72-80. 2012.

[24] Wang, Y., Tang, C.Y., and Zhang, H., "Hepatoprotective effects of kaempferol-3-O-rutinoside and kaempferol-3-O-glucoside from Carthamus tinctorius $\mathrm{L}$. on $\mathrm{CCl}_{4}$-induced oxidative liver injury in mice," Journal of Food and Drug Analysis, 23. 310-317. 2015.

[25] Peng, X., Zhang, G., Liao, Y., and Gong, D., "Inhibitory kinetics and mechanism of kaempferol on $\alpha$-glucosidase," Food Chemistry, 190. 207-215. 2016.

[26] Choung, M.G., Choi, B.R., An, Y.N., Chu, Y.H., and Cho, Y.S., "Anthocyanin profile of Korean cultivated kidney bean (Phaseolus vulgaris L.)," Journal of Agricultural and Food Chemistry, 51. 7040-7043. 2003.

[27] Han, K.H., Okada, T.K., Seo, J.M., Kim, S.J., Sasaki, K., Shimada, K., and Fukushima, M., "Characterisation of anthocyanins and proanthocyanidins of adzuki bean extracts and their antioxidant activity," Journal of Functional Foods, 14. 692-701. 2015. 